\title{
Desempenho no teste de atenção dividida como resultado da idade das pessoas
}

\author{
Performance in the divided attention \\ test as a function of people's age
}

Fabián Javier Marín RUEDA

\begin{abstract}
Resumo
A literatura sobre atenção assinala acréscimo do desempenho atencional até a idade de adulto jovem, seguido de perdas significativas decorrentes do processo de envelhecimento. Dentro desse contexto, o objetivo desta pesquisa foi verificar evidência de validade em função da variável idade para o Teste de Atenção Dividida, partindo da hipótese de que ao aumento da idade corresponderia uma diminuição da capacidade de dividir a atenção. Participaram da pesquisa 878 indivíduos dos Estados da Bahia e Sergipe, sendo eles estudantes universitários ou pessoas que passaram por avaliação psicológica para motorista, com idade entre 18 e 72 anos $(M=26,74, D P=8,14)$. Do total, 51,82\% eram homens. $O$ instrumento foi aplicado coletivamente no Estado de Sergipe, e de forma individual na Bahia. Os resultados mostraram correlação negativa e significativa entre a pontuação do Teste de Atenção Dividida e a idade das pessoas. A análise de variância mostrou diferenças significativas entre as idades, permitindo agrupar três faixas etárias, diferenciadas pela prova de Tukey. Com base nos resultados concluiu-se por evidência de validade para o Teste de Atenção Dividida com base na diferenciação das idades.
\end{abstract}

Unitermos: Atenção dividida. Testes psicológicos. Validade do teste.

\begin{abstract}
Literature points to incremental performance in attention up untilyoung adulthood, followed by significant losses arising from the aging process. In this context, the aim of this research was to ascertain proof of validity based on the age variable for the Divided Attention Test, based on the hypothesis that an increase in age would correspond to a decrease in the ability to divide attention. A total of 878 individuals took part from the states of Bahia and Sergipe and were undergraduates or individuals and that had undergone psychological assessment of drivers. Ages varied from 18 to 72 years old ( $M=26.74, S D=8.14)$. Men comprised 51.82\% of the total. The instrument was applied collectively in the state of Sergipe, and individually in Bahia. Results showed significant, negative correlation between the Divided Attention Test scores and people's age. Analysis of variance showed significant differences between ages and, based on this, three age groups were formed that were differentiated by Tukey's test. Based on the results, it was concluded that there was evidence of validity for the Divided Attention Test based on age difference.
\end{abstract}

Uniterms: Divided attention. Psychological test. Test validety.

O processo atencional tem sido muito investigado na psicologia, neuropsicologia, neurociência e fisiologia, devido a sua relevância em diversos contextos, como a escola, o trabalho e o trânsito. A atenção tem sido apontada como um aspecto de destaque em outros processos cognitivos, como a memória e a psicomotri-

$\boldsymbol{\nabla \nabla \nabla \nabla}$

1 Universidade São Francisco, Programa de Pós-Graduação Stricto Sensu em Psicologia. R. Alexandre Rodrigues Barbosa, 45, Centro, 13251-900, Itatiba, SP, Brasil. E-mail: <marinfabian@yahoo.com.br>. 
cidade (Kallus, Schmitt \& Benton, 2005; Kouststaal, Schacter \& Brenner, 2001; Park \& Smith, 1989), assim como por sua relação com psicopatologias como Alzheimer, Parkinson e esquizofrenia (Bondi et al., 2002; Briand, Hening, Poinzner \& Sereno, 2001; Digirolamo \& Posner, 1996; Fan, McCandliss, Sommer, Raz \& Posner, 2002), além de sua relação com outros construtos da psicologia.

Por ser um processo multideterminado, sua definição e classificação apresentam algumas dificuldades. De forma geral, a atenção pode ser definida como o fenômeno que administra a grande quantidade de informações disponibilizadas ao organismo por meio dos sentidos, memória e outros processos cognitivos, originando-se de uma predisposição do cérebro para responder a determinados estímulos em detrimento de outros (Brandão, 1995; Gazzaniga, Ivry \& Mangun, 2006; Sternberg, 2000). Complementando essas informações, Goldberg, Gold e Braff (1991) apontaram que, em virtude da importância da atenção em diversas operações mentais, ela poder ser considerada como um dos componentes mais importantes no grupo das funções cognitivas. Por sua vez, para Silva (1999), a capacidade atencional é a função cerebral responsável pela escolha dos estímulos que representam o foco de maior interesse em um dado momento, possuindo grande importância para a realização de várias tarefas.

As reflexões acerca da atenção devem fazer referência a seus diferentes tipos, uma vez que, de acordo com sua função ou operacionalização, diversos critérios podem ser utilizados para seu aferimento. Segundo Lent (2001), a atenção pode ser dividida em dois componentes: a atenção explícita, referente aos processos conscientes, como a fixação visual intencional num foco da atenção; e a atenção implícita, relativa aos processos não conscientes, como a atenção a estímulos visuais que não são o alvo e estão na periferia do campo visual. Em relação à atenção explícita, Luria (1979) acrescenta que a seleção dos estímulos e o direcionamento da atividade mental são mediados também pela importância e interesse por determinada tarefa em detrimento de outras.

Deve ser ressaltado que, embora as teorias e definições sobre a atenção sejam amplas, não há um consenso entre os pesquisadores em relação à melhor definição para o construto, sendo que muitas vezes

252 aplicam-se definições operacionais. Nesse sentido, pode- -se fazer referência a Brickenkamp (2004), que observa que, enquanto na Alemanha o termo mais utilizado é "atenção seletiva", nos Estados Unidos são muito comuns os termos "atenção seletiva"e "atenção sustentada". Ainda, autores como Zillmer e Spiers (1998) apontam que muitas vezes a atenção seletiva é definida também como atenção concentrada. Por sua vez, Lezak (1995) afirma que um teste como o d2, que se propõe avaliar a atenção concentrada, na Alemanha é conhecido como "teste de concentração" ou "teste de atenção seletiva", enquanto nos Estados Unidos recebe o nome de "teste de amplitude atencional", "teste de atenção seletiva" ou "teste de atenção sustentada".

Com base nisso, pode-se dizer que atualmente há no Brasil a aceitação e definição de quatro grandes tipos de atenção, quais sejam: atenção dividida, alternada, concentrada e sustentada. Neste estudo será abordado principalmente o conceito de atenção dividida. Segundo Sisto, Noronha, Lamounier, Bartholomeu e Rueda (2006), a atenção dividida é avaliada, tradicionalmente, solicitando-se que a pessoa tenha como foco pelo menos dois estímulos (estímulos-meta). 0 distrator usado em uma situação como essa, via de regra, são outros estímulos concorrentes, distribuídos entre os estímulo-meta. Por sua vez, para Chan (1999), quando os sujeitos são estimulados em duas situações distintas, a atenção é dividida entre os estímulos, alocando-se recursos mentais para cada um deles.

Como foi mencionado, da mesma forma que o construto da atenção apresenta várias definições e operacionalizações, ele também foi relacionado a diversas variáveis ao longo dos estudos realizados. Nesse contexto, dentre os temas de pesquisa sobre atenção, encontram-se aqueles relacionados ao seu desenvolvimento ao longo do ciclo da vida. Papalia e Olds (2000) e Sanchez-Gil e Perez-Martinez (2008) afirmam que a literatura específica sobre o desenvolvimento cognitivo assinala acréscimos, em termos de desempenho, até a idade de adulto jovem, seguidos de perdas significativas decorrentes do processo de envelhecimento. No caso da atenção, essas perdas ocorreriam principalmente quando se fala da atenção explícita.

Pesquisando a relação entre idade e atenção, Pesce, Guidetti, Baldari, Tessitore e Capranica (2005) realizaram uma pesquisa com a participação de três grupos com 14 sujeitos cada, cujas idades variaram de 12 a 15 anos, de 24 a 38 anos e de 60 a 75 anos, respectivamente. A tarefa consistiu em localizar um alvo pre- 
viamente definido após uma dica visual. Em seus resultados, os autores verificaram que os idosos apresentaram, sistematicamente, um desempenho pior do que os adultos jovens e os adolescentes, mas não houve diferenças entre esses dois últimos grupos. Nesse sentido, Pesce et al. (2005) sugeriram que o pior desempenho verificado nos idosos poderia ser resultado de deficiências orgânicas decorrentes do envelhecimento.

Essa afirmação já tinha sido realizada por Hawkins, Kramer e Capaldi (1992), ao investigarem a relação entre idade e processo atencional, por meio de duas tarefas de atenção (dividida e alternada), ambas apresentando medidas visuais e auditivas que foram apresentadas separadamente. Participaram da pesquisa dois grupos com 14 sujeitos cada, um entre 20 e 35 anos, e outro entre 67 e 74. Quando comparado o tempo de reação dos grupos, os autores observaram que os adultos jovens foram sistematicamente mais rápidos que os idosos.

Essa possível diferença na capacidade atencional entre pessoas mais jovens e idosos não parecia ser restrita a uma ou outra atividade da vida diária, mas podia estar presente em diversas situações, dentre as quais a condução de veículos automotores. Algumas pesquisas acerca do tema sugerem que deficits na atenção visual de motoristas mais velhos estariam associados a habilidades mais pobres de condução e, consequentemente, a maior risco de acidentes (Ball \& Owsley, 2003; Tielsch, Sommer \& Witt, 1990; Underwood, 2007). Por sua vez, esses autores sugerem que tais perdas poderiam ser compensadas por comportamentos mais seguros e pela experiência, gerando vantagens para os motoristas mais velhos quando comparados aos mais jovens.

Quanto ao aspecto visual, de uma forma geral, e sua relação com o ato de dirigir, alguns dos trabalhos mais influentes foram realizados por Burg (1967; 1968). Seus estudos analisaram dados de 17500 motoristas dos Estados Unidos, indicando que para os motoristas jovens e de meia-idade não haveria nenhuma relação entre desempenho visual pobre e índices de acidente de trânsito. Já em relação aos motoristas idosos, a deterioração da acuidade visual mostrou correlações positivas e significativas com o envolvimento em acidentes de trânsito. Corroborando essa informação, Owsley (1994) informou que motoristas mais velhos, com uma diminuição de aproximadamente $40 \%$ do campo visual, eram mais propensos a se envolver em acidentes de trânsito do que indivíduos mais novos com as mesmas características visuais.
H. C. Lee, A. H. Lee e Cameron (2003) avaliaram a velocidade de tempo de reação em acidentes de trânsito com motoristas idosos, num simulador motriz, e informaram que a habilidade de atenção visual de pessoas mais velhas seria decisiva para predizer o envolvimento ou não em acidentes. Ainda no que diz respeito à atenção e à população de motoristas mais velhos, a pesquisa de Sanders (1970) avaliou medidas de atenção seletiva, dividida e velocidade de processamento em idosos que possuíam carteira de habilitação. O autor concluiu que o envolvimento em acidentes de trânsito nessa população estaria associado à diminuição da atenção de uma forma geral. Por sua vez, ao pesquisar especificamente a atenção dividida, Owsley, Ball e McGwin (1998) verificaram que os motoristas idosos com problemas de atenção dividida apresentavam uma probabilidade 2,2 maior de se envolver em acidentes do que aqueles idosos sem problemas de atenção.

Campagne, Pebayle e Muzet (2004) afirmaram que, ao dirigir por muito tempo de forma monótona ou à noite, a maior parte dos motoristas mostraria progressivamente perda da atenção e sinais de fadiga visual. Destacaram que a capacidade para manter um desempenho adequado ao dirigir seria comumente afetada em função da idade do motorista. Dessa forma, os autores propuseram um estudo para verificar os efeitos da fadiga e da idade dos motoristas sobre seu desempenho na direção. Foram estudados 46 motoristas, divididos em três categorias de idade, de 20 a 30 anos, de 40 a 50 e de 60 a 70. Foram medidos os erros dos motoristas quanto ao número de incidentes e desvios em alta velocidade, enquanto percorriam mais de $300 \mathrm{~km}$ numa via, de madrugada. A evolução do nível de atenção foi avaliada por meio do eletroencefalograma. Nos indivíduos mais velhos, foi verificado que o declínio no desempenho de dirigir estava, de fato, associado com o declínio da capacidade atencional. Por outro lado, nos motoristas jovens e de meia idade, a piora no nível de atenção apresentou correlação com o aumento na gravidade dos erros.

As pesquisas estrangeiras relatadas até o momento mostram que o estudo da atenção de idosos em situação de condução tem acontecido de diversas formas, sendo que todos os autores citados parecem concordar quanto à relação inversa entre idade e desempenho atencional. Também, praticamente, não há relatos de testes psicológicos que objetivem avaliar a atenção 
desse tipo de população ao dirigir veículos automotores, sendo as avaliações muitas vezes realizadas em simuladores.

No Brasil, a avaliação por meio de simuladores ainda parece um pouco distante, haja vista que praticamente toda avaliação psicológica para possíveis condutores de veículos automotores é realizada por meio de testes psicológicos, sendo a maior parte deles gráficos. Nesse sentido, nos últimos anos a literatura científica da área tem se preocupado bastante em atribuir evidências de validade para testes psicológicos utilizados no contexto do trânsito, inclusive relacionando-as com a variável idade.

Dentro desse contexto, Rueda, Noronha, Sisto e Bartholomeu (2008) objetivaram verificar evidência de validade em relação à idade para o Teste de Atenção Sustentada (AS). Participaram 127 indivíduos que passaram pelo processo de avaliação psicológica para obtenção da Carteira Nacional de Habilitação (CNH) no Estado de Minas Gerais, e nos resultados foram verificadas correlações negativas e significativas entre as medidas de Concentração e de Velocidade com Qualidade do AS com a variável idade ( $r=-0,38$ e $r$-=0,37, respectivamente). Ainda, os autores realizaram uma análise de variância com seis faixas etárias, quais sejam indivíduos de 18 a 19 anos; de 20 a 27; de 28 a 36; de 37 a 41; de 42 a 55 anos; e com mais de 56 anos. Os resultados mostraram que ao aumento da idade correspondia uma diminuição da capacidade de atenção sustentada, sugerindo a criação de normas específicas para cada faixa etária e concluindo pela verificação de evidência de validade de construto com base na análise das idades.

Por sua vez, estudo similar foi realizado por Noronha, Sisto, Rueda e Bartholomeu (2008) para o Teste de Atenção Dividida (AD). O instrumento foi aplicado em 369 candidatos à obtenção de CNH, com idade entre 18 e 73 anos. Os resultados também mostraram correlações negativas entre as medidas de Velocidade com Qualidade e de Concentração com a idade, sugerindo que as pontuações diminuíam conforme aumentava a idade. Na análise dos autores, foram encontrados seis grupos etários em ambas as medidas, com faixas de 18 a 19 anos, de 20 a 27, de 28 a 36, de 37 a 41, de 42 a 55; e de 56 anos a 73. Dessa forma, os autores também concluíram que a capacidade de atenção dividida diminuiria com o passar da idade e que normas específicas deve-

254 riam ser criadas.
Estudando diferenças em função da idade para o Teste de Atenção Concentrada (TEACO-FF), Rueda e Sisto (2009) realizaram uma pesquisa com 1058 pessoas dos Estados da Bahia e Sergipe, com idade entre 18 e 61 anos. A análise realizada pelos autores separou os resultados em três faixas etárias: até os 27 anos, entre 28 e 35 anos; e com 36 anos ou mais. A análise de variância e a prova de Tukey mostraram diferenças estatisticamente significativas entre os grupos, sendo que as pessoas mais novas apresentaram maiores escores em atenção concentrada, enquanto os mais velhos tiveram os piores desempenhos.

Por fim, no estudo de Rueda e Castro (2009), o objetivo foi procurar evidência de validade, baseada na variável idade para o Teste de Atenção Alternada (TEALT). Participaram 798 indivíduos dos Estados da Bahia e Sergipe, com idades entre 18 e 72 anos. Os resultados evidenciaram correlação negativa entre a pontuação do TEALT e a idade, sendo formadas três faixas etárias, que se diferenciaram no desempenho no teste pela análise de variância $[F(2,797)=60,01, p<0,001]$. Dessa forma, concluiu-se por evidência de validade para o TEALT em função da idade, sendo que ao aumento da mesma correspondeu uma diminuição da capacidade de atenção alternada. Ao autores diferenciaram faixas etárias até os 22 anos, dos 23 aos 32 anos, e de 33 anos ou mais.

Dentro desse contexto, e tendo como base os estudos nacionais apresentados sobre o tema, este estudo se propôs a verificar evidência de validade em função da variável idade para o Teste de Atenção Dividida - TEADI (Rueda, 2010). A hipótese inicial foi que as pessoas poderiam ser diferenciadas em função da idade, sendo que ao aumento da idade corresponderia uma diminuição da capacidade de dividir a atenção.

\section{Método}

\section{Participantes}

Participaram deste estudo 878 pessoas dos Estados de Bahia e Sergipe, com idade de 18 a 72 anos (Média - $M=26,74$, Desvio-Padrão - DP=8,14). Quanto ao sexo, 455 (51,82\%) eram homens e 423 (48,18\%) mulheres. Do Estado da Bahia, participaram da amostra 488 candidatos que passaram pelo processo de avaliação psicológica pericial para obtenção, renovação ou mudança de categoria da $\mathrm{CNH}$, sendo 332 (68\%) homens e 
156 (32\%) mulheres. No que se refere à idade, a média foi de 28,03 anos (DP=8,77), sendo a mínima 18 e a máxima 72. Já do Estado de Sergipe participaram 390 estudantes de uma universidade particular da cidade de Aracaju, sendo 123 (31,5\%) homens e 267 (68,5\%) mulheres. A média de idade foi de 25,12 anos ( $D P=6,96)$, variando de 18 a 60. Vale destacar que, dos participantes desse Estado, 271 pessoas possuíam $\mathrm{CNH}$, em diferentes categorias.

Deve ser destacado que a maior concentração da amostra (53,3\%) ficou entre os 20 e os 27 anos. Dos 28 aos 40 anos observou-se uma diminuição das idades estudadas, sendo que, a partir dos 40 anos, essa diminuição foi consideravelmente mais acentuada. Levando em consideração que os participantes foram estudantes universitários e candidatos à $\mathrm{CNH}$, a falta de um número razoável de sujeitos representativo de cada uma das idades pareceria ser justificável.

\section{Instrumento}

Foi aplicado TEADI, que fornece uma medida referente à capacidade de uma pessoa dividir a atenção. Para construção do TEADI foram realizados alguns estudos, até se chegar à forma final que o teste possui, tanto na quantidade de estímulos como nos símbolos abstratos que a pessoa deveria procurar e assinalar ou não.

Para isso, partiu-se da afirmação de Alchieri, Lunkes e Zimmer (2002), que relataram que um dos primeiros instrumentos de medida para avaliar a atenção foi o Teste de Bourdon, de 1895, consistente em marcar todas as letras "A" (estímulo-alvo) da folha de resposta. No caso específico desse instrumento, foi verificado que a distribuição das letras na página não era uniforme e que a execução da prova era extremamente influenciada pela experiência anterior da pessoa e seu nível de escolaridade. Com base nesses aspectos, o teste foi modificado por uma página na qual não eram verificadas palavras escritas, e sim letras distribuídas irregularmente. Finalmente, essas letras foram substituídas por figuras (quadrinhos) que se caracterizavam por ter um pequeno segmento que se sobressaía de um de seus ângulos ou lados. Segundo Hiltmann (1962), essa substituição eliminou os fatores de familiaridade com as letras e de escolaridade. Deve ser destacado que o teste serviu como uma base e propiciou o estudo e construção de muitos instrumentos posteriores para avaliar a atenção. Dessa forma, para a construção do TEADI foram selecionados três símbolos que o participante deveria pro- curar e marcar em cada linha do teste. A seguir, três psicólogos experientes na área analisaram o formato que os estímulos deveriam possuir, decidindo que eles deveriam ser abstratos, tanto no que se refere aos estímulos-alvo quanto aos distratores. Ainda, a localização de cada estímulo foi realizada por meio de sorteio, para evitar possíveis vieses.

Na sua totalidade, o instrumento possuía 450 estímulos, distribuídos em 30 linhas com 15 estímulos cada uma. O resultado obtido no TEADI pôde ser calculado com base nos estímulos que a pessoa deveria marcar e marcou, subtraindo-se os erros (estímulos que não deveriam ser marcados e foram) e omissões (estímulos-alvo que não foram marcados). O tempo de aplicação foi de 5 minutos; para se chegar a esse critério, o instrumento foi aplicado sem tempo limite, em aproximadamente 100 indivíduos, obtendo-se como tempo médio de realização 6'08", até fixar-se finalmente o tempo de 5 minutos.

\section{Procedimentos}

Num primeiro momento, estabeleceu-se contato com a instituição de ensino superior de Sergipe e com as clínicas credenciadas pelo DETRAN-BA, sendo explicados os propósitos da pesquisa. Após aprovação pelo Comitê de Ética em Pesquisa da Universidade São Francisco (protocolo no 0189.0.142.000-09), procedeu-se à coleta de dados, tendo todos os participantes assinado o Termo de Consentimento Livre e Esclarecido. No caso dos estudantes universitários de Sergipe, a aplicação foi realizada de forma coletiva em sala de aula, com um número máximo de estudantes de 30 por aplicação. Já na pesquisa empreendida na Bahia, as aplicações foram realizadas individualmente nas clínicas de avaliação psicológica credenciadas pelo DETRAN-BA.

Durante a aplicação, as instruções foram apresentadas de forma padronizada, tanto na Bahia quanto em Sergipe. Assim, após o preenchimento dos dados pessoais pelos participantes, procedeu-se às instruções:

Este é um teste de atenção. No verso desta folha vocês deverão assinalar com um traço todos os desenhos que forem iguais aos modelos que se encontram na parte superior da folha (os desenhos foram mostrados). Vocês deverão começar da esquerda para a direita e voltar da direita para a esquerda de forma contínua, sempre seguindo as setas da folha do teste (as setas foram mostradas). Caso erre, circule e assinale o item correto. 
Vocêterá 5 minutos para realizar o teste. Lembre-se que este é um teste de atenção. Portanto, concentre-se e procuremanter seuritmo de trabalho. Evitese distraircom outras coisas e fique calmo.

Deve ser destacado que, no caso da aplicação realizada no Estado da Bahia, por ter sido feita de forma individual, as instruções foram reproduzidas no singular.

\section{Resultados}

Na análise dos dados, num primeiro momento foram calculadas as estatísticas descritivas do TEADI. Posteriormente, foi feita a correlação entre a idade do participante e a pontuação obtida, para serem comparadas as possíveis faixas etárias formadas em relação ao desempenho de atenção dividida. Destaca-se que a análise foi realizada para cada Estado de forma separada, sendo os resultados apresentados em função da faixa etária e do sexo do participante.

Em relação ao desempenho no TEADI, a pontuação variou de -126 a $180(M=108,61, D P=43,62)$. A mediana foi 113 ea moda 128 . Foi muito pequena $(1,6 \%)$ a quantidade de pessoas que apresentaram pontuações negativas, ou seja, cometeram mais erros e/ou omissões do que acertos. Houve, também, uma concentração de pontuações entre 80 e 140 pontos, congregando 457 dos participantes (52,83\%). O número de pessoas que atingiu a pontuação máxima (180 pontos) representou 0,9\% da amostra (8 indivíduos).

Em continuação, foi realizada a prova de correlação de Pearson, para investigar a relação existente entre o TEADI e a idade. O resultado mostrou uma correlação de $-0,37$ ( $p<0,001)$, o que indica que ao aumento da idade correspondeu uma diminuição da atenção dividida.

Ainda foram estudadas as variações das pontuações médias no TEADI em função das diferentes idades, conforme ilustra a Figura 1. Pode-se observar que, em que pese a variação nas médias ao longo das idades, existe uma diminuição da atenção dividida com o aumento da mesma.

Deve ser ressaltado que a variabilidade observada fica mais acentuada a partir dos 42 anos, o que pode ser atribuído ao menor número de participantes. Dessa forma, procurou-se agrupar as idades em faixas etárias que melhor diferenciassem essa variável.

Assim, após várias análises, as faixas etárias for-

256 madas que apresentaram o maior valor de F foram: até os 25 anos, de 26 a 35 anos, e de 36 anos ou mais. Na faixa etária até 25 anos ficaram agrupadas 497 pessoas (57,46\%); dos 26 a 35 anos, 253 pessoas (29,25\%); e com 36 anos ou mais, 115 pessoas (13,29\%).

O resultado da análise de variância dessas faixas etárias mostrou diferenças estatisticamente significativas $[F(2,864)=53,57, p<0,001]$. Com a finalidade de verificar a pontuação média de quais faixas etárias poderiam explicar tais diferenças, realizou-se a Prova de Tukey, cujos resultados podem ser observados na Tabela 1.

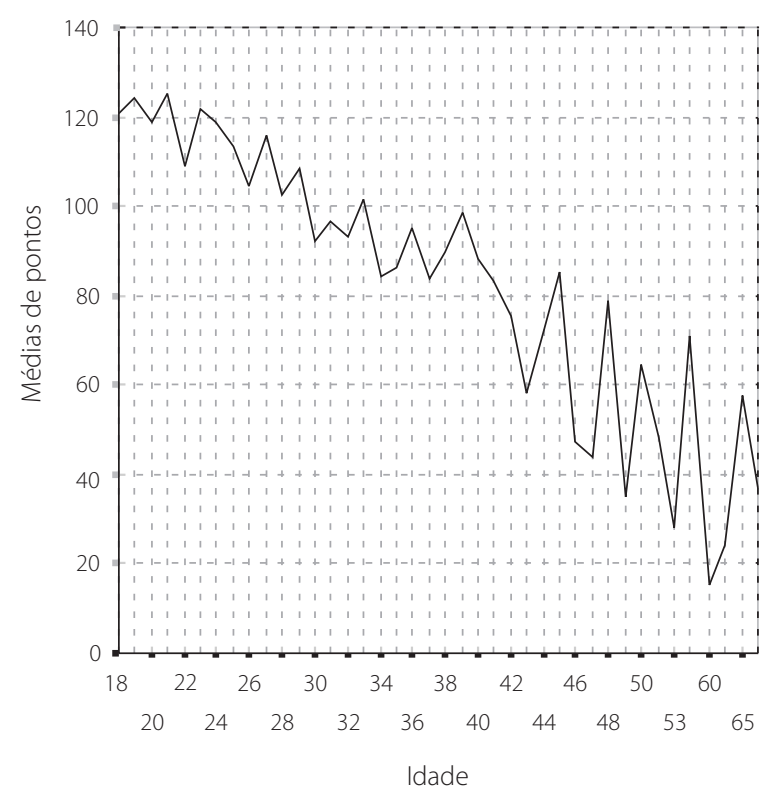

Figura 1. Média de pontos no TEADI e idade dos participantes.

Tabela 1. Subgrupos de faixas etárias formados pela prova de Tukey para o TEADI.

\begin{tabular}{lcccc}
\hline Faixas etárias & $\mathrm{n}$ & \multicolumn{3}{c}{ Subgrupo para alfa $=0,05$} \\
\cline { 3 - 5 } & & 1 & 2 & 3 \\
\hline 36 anos ou mais & 115 & 77,17 & & \\
26 a 35 anos & 253 & & 101,92 & \\
Até 25 anos & 497 & & & 119,29 \\
\hline$p$ & & 1,000 & 1,000 & 1,000 \\
\hline
\end{tabular}

Tabela 2. Subgrupos de faixas etárias no Estado da Bahia, formados pela prova de Tukey para o TEADI.

\begin{tabular}{lcccc}
\hline \multirow{2}{*}{ Faixas etárias } & $\mathrm{n}$ & \multicolumn{3}{c}{ Subgrupo para alfa $=0,05$} \\
\cline { 2 - 5 } & & 1 & 2 & 3 \\
\hline 36 anos ou mais & 82 & 77,24 & & \\
26 a 35 anos & 169 & & 101,08 & \\
Até 25 anos & 228 & & & 116,16 \\
\hline$p$ & & 1,000 & 1,000 & 1,000 \\
\hline
\end{tabular}


A Tabela 1 permite observar que os três grupos etários foram diferenciados, evidenciando que quanto mais velhas as pessoas, menor a pontuação em atenção dividida. Ainda procurou-se verificar se as faixas etárias diferenciadas na amostra total eram mantidas em função de cada um dos estados analisados. No Estado da Bahia, a configuração das faixas etárias mostrou que até os 25 anos houve 228 pessoas (47,60\%); dos 26 aos 35 anos, 169 indivíduos (35,28\%); e na faixa etária de 36 anos ou mais, 82 pessoas (17,12\%). A análise de variância (ANOVA) mostrou diferenças significativas $[F(2,478)=31,04$, $p<0,001]$. Na Tabela 2 é apresentada a análise da Prova de Tukey para explicar as diferenças encontradas.

Na Tabela 2, observa-se que as faixas etárias definidas também foram diferenciadas na amostra da Bahia, em que as pessoas mais novas obtiveram o melhor desempenho. Quanto ao Estado de Sergipe, a faixa etária até 25 anos teve 269 indivíduos (69,69\%); de 26 a 35 anos, 84 pessoas (21,76\%); e de 36 anos ou mais, 33 pessoas (8,55\%). A ANOVA $[F(2,385)=18,49, p<0,001]$ mostrou diferenças estatisticamente significativas. A prova de Tukey é apresentada na Tabela 3 para analisar essas diferenças encontradas.

No Estado de Sergipe também foi verificado que as três faixas etárias foram diferenciadas, mantendo a

Tabela 3. Subgrupos de faixas etárias no Estado de Sergipe formados pela prova de Tukey para o TEADI.

\begin{tabular}{lcccc}
\hline \multirow{2}{*}{ Faixas etárias } & $\mathrm{n}$ & \multicolumn{3}{c}{ Subgrupo para alfa $=0,05$} \\
\cline { 3 - 5 } & & & 1 & 2 \\
\hline 36 anos ou mais & 33 & 76,97 & \\
26 a 35 anos & 84 & & 103,63 & \\
Até 25 anos & 269 & & & 121,94 \\
\hline$p$ & & 1,000 & 1,000 & 1,000 \\
\hline
\end{tabular}

Tabela 4. Estatísticas por sexo da amostra total e das faixas etárias no TEADI.

\begin{tabular}{llrccc}
\hline & Sexo & $M$ & $D P$ & $t$ & $p$ \\
\hline Amostra total & Masculino & 105,72 & 43,57 & & \\
& Feminino & 111,73 & 43,50 & $-1,73$ & 0,053 \\
\multirow{2}{*}{ até 25 anos } & Masculino & 120,87 & 35,42 & & \\
& Feminino & 117,96 & 41,20 & 0,84 & 0,403 \\
de 26 a 35 anos & Masculino & 99,00 & 42,67 & & \\
& Feminino & 106,71 & 40,32 & $-1,42$ & 0,156 \\
36 anos ou mais & Masculino & 69,03 & 46,13 & & \\
& Feminino & 87,74 & 52,03 & $-1,74$ & 0,054 \\
\hline
\end{tabular}

M: média; DP: desvio-padrão. mesma configuração apresentada na amostra total e na Bahia. Por fim, a pontuação do TEADI na amostra total e nas faixas etárias foi comparada em relação ao sexo dos participantes. A Tabela 4 mostra os resultados dessa análise.

Na Tabela 4, observa-se que, na amostra total e nas faixas etárias de 26 a 35 anos e de 36 anos ou mais, as mulheres obtiveram uma pontuação maior que os homens no TEADI. Já entre os participantes que se encontravam na faixa etária até os 25 anos, o sexo masculino apresentou uma média maior do que o feminino. Porém, nenhuma dessas diferenças foi estatisticamente significativa, o que indica que elas podem ser atribuídas ao acaso.

\section{Discussão e Considerações finais}

Considerando o objetivo proposto neste estudo (verificar evidência de validade para o TEADI em função da idade das pessoas - Rueda, 2010), bem como a hipótese de que as pessoas se diferenciariam em função dessa variável, numa relação inversa entre idade e capacidade atencional, deve-se lembrar da afirmação de autores como Papalia e Olds (2000) de que a capacidade atencional mantida voluntariamente passaria por um declive significativo, natural do envelhecimento.

Nesse sentido, esta pesquisa verificou que ao aumento da idade correspondeu uma diminuição da capacidade de dividir a atenção $(r=-0,37)$. Comparando os resultados com a literatura sobre o tema, percebe-se que os achados vão ao encontro das pesquisas de Noronha et al. (2008), Rueda et al. (2008), Rueda e Castro (2009) e Rueda e Sisto (2009), que verificaram uma diminuição da capacidade atencional com o passar da idade, independentemente do tipo de atenção avaliado.

Ainda, este estudo diferenciou três faixas etárias: pessoas com até 25 anos; de 26 a 35 anos; e de 36 anos ou mais - resultado que corrobora os achados das pesquisas anteriormente citadas. Nesse sentido, deve ser ressaltado que, muito embora a investigação de Rueda et al. (2008) tenha verificado a existência de seis faixas etárias no Teste de Atenção Sustentada (AS), diferenciou apenas três grupos pela prova de Tukey.

Esse mesmo fato foi evidenciado na pesquisa de Noronha et al. (2008), ao estudarem o Teste de Atenção 
Dividida. Ou seja, em que pese a formação de seis faixas etárias, apenas três grupos etários puderam ser considerados como sendo totalmente diferenciados em função da atenção dividida avaliada pelo AD.

Quanto às pesquisas de Rueda e Castro (2009) e Rueda e Sisto (2009), os dados mostraram a separação de três faixas etárias, totalmente diferenciadas na prova de Tukey. No caso de Rueda e Sisto (2009), os grupos etários dividem-se em faixas de até 27 anos, de 28 a 35 anos, e de 36 anos ou mais. Por sua vez, na pesquisa de Rueda e Castro (2009) diferenciaram-se os grupos etários de até 22 anos, de 23 a 32 anos, e de 33 anos ou mais.

Nesse sentido, esta pesquisa parece evidenciar ainda mais a relação existente entre a capacidade atencional e sua diminuição com o passar da idade: até aproximadamente os 25 anos haveria um aumento progressivo da atenção, seja ela dividida, alternada, sustentada ou concentrada; passando por uma leve diminuição até aproximadamente os 35 anos; diminuição essa que pareceria ser mais acentuada, sistematicamente, a partir dessa idade.

No entanto, deve ser ressaltado que a presente investigação não contou com uma participação representativa da terceira idade (pessoas acima de 60 anos). Assim, a amostra desta pesquisa pode ser considerada representativa até os 40 anos, pois a partir dessa idade houve uma diminuição considerável das idades participantes.

De forma geral, este estudo apresentou claramente as diferenças existentes em função das faixas etárias, o que pode ser considerado evidência de validade de construto com base na comparação entre as idades para a atenção dividida avaliada pelo TEADI. Isso sugere o estabelecimento de normas específicas para a interpretação dos resultados desse instrumento em função da idade.

Ainda no que se refere às faixas etárias estudadas, a investigação também procurou verificar se elas eram diferenciadas em função dos Estados participantes da pesquisa. Na Bahia, verificou-se que as três faixas etárias foram diferenciadas, tendo as pessoas mais novas obtido o melhor desempenho. Dessa forma, seria necessária a criação de normas em função das faixas etárias para esse Estado. Por sua vez, em Sergipe também foi verificado que as três faixas etárias foram diferenciadas, mantendo a mesma configuração apresentada na amos- tra total e na Bahia. Assim, para aquele Estado também deveriam ser criadas normas específicas em função das faixas etárias.

Finalmente, foi observado que essas faixas etárias não foram diferenciadas significativamente em função do sexo, quando estudada a amostra total da pesquisa. Desse fato pode ser destacado que não seria necessária a criação de normas separadas em função do sexo para oTEADI.

Especificamente no que tange ao contexto do trânsito, é necessário mencionar que não existe consenso entre os pesquisadores quanto à influência da idade no bom ou mau desempenho como motorista. Se há aqueles que acreditam que os idosos apresentam pior desempenho ao dirigir, em razão da capacidade atencional diminuída (Lee et al., 2003; Sanders, 1970), há também aqueles que afirmam que a diminuição dessa capacidade seria compensada por comportamentos seguros e pela experiência (Ball \& Owsley, 2003; Underwood, 2007). Dessa forma, a necessidade de criação de normas específicas para os diferentes testes atencionais em função da idade pode ser considerada um passo inicial e muito importante na avaliação de motoristas no contexto brasileiro. Por outro lado, destaca-se que estudos que vão além desse objetivo são extremamente necessários, como investigações acerca das reais relações entre idade avançada e (bom ou mau) desempenho como motorista.

\section{Referências}

Alchieri, J. C., Lunkes, C. L. K., \& Zimmer, D. (2002). Toulouse-Piéron: atualizações de resultados para o Estado do Rio Grande do Sul. Avaliação Psicológica, 1 (2), 111-118.

Ball, K., \& Owsley, C. (2003). Driving competence: it's not a matter of age. Journal of American Geriatrics Society, 51 (10), 1499-1501.

Bondi, M. W., Chan, A. S., Delis, D. C., Serody, A. B., EbersonShumate, S. C., Salmon, D. P., et al. (2002). Cognitive and neuropathologic correlates of stroop color-word test performance in Alzheimer's disease. Neuropsychology, $16(3), 335-343$.

Brandão, M. L. (1995). Atenção. In M. L. Brandão (Org.), Psicofisiologia (pp.145-154). São Paulo: Atheneu.

Briand, K. A., Hening, W., Poinzner, H., \& Sereno, A. B. (2001). Automatic orienting of visuospatial attention in Parkinson's disease. Neuropsychologia, 39, 1240-1249.

Brickenkamp, R. (2004). d2: test de atención ( $2^{\text {nd }}$ ed.). Madrid: TEA Ediciones. 
Burg, A. (1967). The relationship between vision test scores and driving record: general findings (Report 67-24). Los Angeles: Department of Engineering.

Burg, A. (1968). The relationship between vision test scores and driving record: additional findings (Report 68-27). Los Angeles: Department of Engineering.

Campagne, A., Pebayle, T ., \& Muzet, A. (2004). Correlation between driving errors and vigilance level: influence of the driver's age. Physiological Behaviors, 80 (4), 515-524.

Chan, R. C. K. (1999). Attentional deficits in patients with closed head injury: a further study to the discriminativ validity of the test of evereday attention. Brain Injury, 14 (3), 227-236

Digirolamo, G. J., \& Posner, M. I. (1996). Attention and schizofrenia: a view from cognitive neurosciense. Cognitive Neuropsychiatry, 1 (2), 95-102.

Fan, J., McCandliss, B. D., Sommer, T., Raz, A., \& Posner, M. I. (2002). Testing the efficiency and independence of attention networks. Journal of Cognitive Neuroscience, 14 (3), 340-347.

Gazzaniga, M. S., Ivry, R. B., \& Mangun, G. R. (2006). Neurociência cognitiva: a biologia da mente. Porto Alegre: Artmed.

Goldberg, T. E., Gold, J. M., \& Braff, D. L. (1991). Neuropsychological functioning and time-linked information processing in schizophrenia. Review of Psychiatry, 10, 60-78.

Hawkins, H. L., Kramer, A. F., \& Capaldi, D. (1992). Aging, exercise, and attention. Psychology and Aging, 7 (4), 643-653.

Hiltmann, H. (1962). Compedio de los test psico-diagnósticos. Buenos Aires: Kapelusz.

Kallus, K. W, Schmitt, J. A. J., \& Benton, D. (2005). Attention, psychomotorfunctions and age. European Journal Nutrition, 44 (8), 465-484.

Kouststaal, W., Schacter, D. L., \& Brenner, C. (2001). Dual task demands and gist-based false recognition of pictures in younger and older adults. Journal of Memory and Language, 44 (3), 399-426.

Lee, H. C., Lee, A. H., \& Cameron, D. (2003). Validation of a driving simulator by measuring the visual attention skill of older adult drivers. American Journal of Occupational Therapy, 57 (3), 324-328.

Lent, R. (2001). Cem bilhões de neuronios: conceitos fundamentais de neurociência. São Paulo: Editora Atheneu.

Lezak, M. D. (1995). Neuropsychological assessment ( $3^{\text {rd }}$ ed.). New York: Oxford University Press.

Luria, A. R. (1979). Curso de psicologia geral. Rio de Janeiro: Civilização Brasileira.

Noronha, A. P. P., Sisto, F. F., Rueda, F. J. M., \& Bartholomeu, D. (2008). Evidência de validade desenvolvimental para o teste de atenção dividida. Psico, 39 (4), 492-499.

Oswley, C. (1994). Vision and driving in the elderly. Optometry and Vision Science, 71 (12), 727-735.

Owsley, C., Ball, K., \& McGwin Jr., G. (1998). Visual processing impair-ment and risk of motor vehicle crash among older adults. JAMA, 279 (14), 1083-1088.
Papalia, D. E., \& Olds, S. W. (2000). Desenvolvimento Humano. São Paulo: Pioneira.

Park, D., \& Smith, A.D. (1989). Effects of age and a divided attention tasl presented during encoding and retrieval on memory. Journal of Experimental Psychology: Learning, Memory and Cognition, 15 (6), 1185-1191.

Pesce, C., Guidetti, L., Baldari, C., Tessitore, A., \& Capranica, L. (2005). Effects of aging on visual attentional focusing. Gerontology, 51 (4), 266-276.

Rueda, F. J. M. (2010). Teste de atenção dividida - TEADI. Itatiba: Universidade São Francisco.

Rueda, F. J. M., \& Castro, N. R. (2009). Capacidade atencional: há decréscimo com o passar da idade? Psicologia: Ciências e Profissão, 30 (3). Disponível em <http://pepisic.bvsalud. org/scielo.php>.

Rueda, F. J. M., Noronha, A. P. P., Sisto, F. F., \& Bartholomeu, D. (2008). Evidência de validade de construto para o teste de atenção sustentada. Psicologia: Ciência eProfissão, 28 (3), 498-505.

Rueda, F. J. M., \& Sisto, F. F. (2009). Evidência de validade de construto pela diferenciação das idades para o TEACO-FF. Psicologia: Ciênciase Profissão, 30(3). Disponível em <http://pepisic.bvsalud.org/scielo.php $>$.

Sanchez-Gil, I. Y., \& Perez-Martinez, V. T. (2008). El funcionamiento cognitivo en la vejez: atención y percepción en el adulto mayor. Revista Cubana de Medicina General Integrada, 24 (2), 1-7.

Sanders, A. F. (1970). Some aspects of the selective process in the functional field of view. Ergonomics, 13 (1), 101-117.

Silva, M. F. G. (1999). Estudo comparativo sobre o desempenho em provas de memória e atenção entre mulheres pré- menstruadas, deprimidas e não deprimidas. Dissertação de mestrado não-publicada, Instituto de Psicologia, Universidade de São Paulo.

Sisto, F. F., Noronha, A. P. P., Lamounier, R., Bartholomeu, D., \& Rueda, F. J. M. (2006). Testes de atenção dividida e sustentada: manual. São Paulo: Vetor Editora Psicopedagógica.

Sternberg, R. J. (2000). Psicologia cognitiva. Porto Alegre: Artmed.

Tielsch, J. M., Sommer, A., \& Witt, K. (1990). The baltimore eye survey. Blindness and visual impairment in an American urban population. Archives of Ophthalmology, 108 (2), 286-290.

Underwood, G. (2007). Visual attention and the transition from novice to advanced driver. Ergonomics, 50 (8), $1235-1249$

Zillmer, E. A., \& Spiers, M. V. (1998). Principles of clinical neuropsychology. Pacific Groove: Brooks/Cole.

Recebido em: 16/7/2009

Versão final reapresentada em: 15/7/2010

Aprovado em: 11/3/2011 
